\title{
Curriculum et Coca-Cola : un nouvel emballage change-t-il la saveur? Les concepts de matière, de discipline, de savoir et de connaissance dans le contexte de la réforme du curriculum au Québec
}

\section{Curriculum and Coca-Cola: does new packaging change the flavour? Concepts of subject matter, discipline, know-how and knowledge in the context of Québec's curriculum reform \\ Curriculum y coca-cola : ¿una nueva presentación cambia el sabor? Los conceptos de materia, asignatura, saber y conocimiento en el contexto de la reforma del curriculum en Quebec}

\author{
François Larose, Vincent Grenon, Sébastien Ratté et Mary Pearson
}

Volume 28, numéro 2, automne 2000

Réforme curriculaire et statut des disciplines : quels impacts sur la formation professionnelle à l'enseignement?

URI : https://id.erudit.org/iderudit/1080452ar

DOI : https://doi.org/10.7202/1080452ar

Aller au sommaire du numéro

Éditeur(s)

Association canadienne d'éducation de langue française

ISSN

0849-1089 (imprimé)

1916-8659 (numérique)

Découvrir la revue

Citer cet article

Larose, F., Grenon, V., Ratté, S. \& Pearson, M. (2000). Curriculum et Coca-Cola : un nouvel emballage change-t-il la saveur? Les concepts de matière, de discipline, de savoir et de connaissance dans le contexte de la réforme du curriculum au Québec. Éducation et francophonie, 28(2), 188-215.

https://doi.org/10.7202/1080452ar
Résumé de l'article

Dans cet article, nous procédons à l'analyse comparative de contenu du discours de certains acteurs principaux du processus de réforme curriculaire et de praticiennes et praticiens de l'enseignement primaire au Québec. Les concepts à l'étude, ceux de matière, de discipline, de matière de base ou secondaire ainsi que de savoir, sont mis en perspective en fonction de leur impact sur les fondements épistémologiques du curriculum et de l'enseignement. Nous terminons l'article en interrogeant la pertinence du recours à un discours qui se veut constructiviste si les concepts qui fondent le discours en éducation sont définis selon une épistémologie réaliste dont l'expression curriculaire ne peut être que de type néobéhavioriste.

Tous droits réservés ( $\odot$ Association canadienne d'éducation de langue française, Ce document est protégé par la loi sur le droit d'auteur. L’utilisation des 2000 services d'Érudit (y compris la reproduction) est assujettie à sa politique d'utilisation que vous pouvez consulter en ligne.

https://apropos.erudit.org/fr/usagers/politique-dutilisation/ 


\section{Curriculum et Coca-Cola : un nouvel emballage change- t-il la saveur? Les concepts de matière, de discipline, de savoir et de connaissance dans le contexte de la réforme du curriculum au Québec}

François LAROSE

Université de Sherbrooke, Québec, Canada

Vincent GRENON

Université de Sherbrooke, Québec, Canada

Sébastien RATTÉ

Université de Sherbrooke, Québec, Canada

Mary PEARSON

Université de Sherbrooke, Québec, Canada 


\title{
RÉSUMÉ
}

Dans cet article ${ }^{1}$, nous procédons à l'analyse comparative de contenu du discours de certains acteurs principaux du processus de réforme curriculaire et de praticiennes et praticiens de l'enseignement primaire au Québec. Les concepts à l'étude, ceux de matière, de discipline, de matière de base ou secondaire ainsi que de savoir, sont mis en perspective en fonction de leur impact sur les fondements épistémologiques du curriculum et de l'enseignement. Nous terminons l'article en interrogeant la pertinence du recours à un discours qui se veut constructiviste si les concepts qui fondent le discours en éducation sont définis selon une épistémologie réaliste dont l'expression curriculaire ne peut être que de type néobéhavioriste.

\section{ABSTRACT}

Curriculum and Coca-Cola: does new packaging change the flavour? Concepts of subject matter, discipline, know-how and knowledge in the context of Québec's curriculum reform

\author{
François LAROSE \\ Universitéy of Sherbrooke, Quebec, Canada \\ Vincent GRENON \\ Universitéy of Sherbrooke, Quebec, Canada \\ Sébastien RATTÉ \\ Universitéy of Sherbrooke, Quebec, Canada \\ Mary PEARSON \\ Universitéy of Sherbrooke, Quebec, Canada
}

In this article ${ }^{1}$, we present the results of a comparative analysis of some documents published by various organisms involved in the curricular reform that is undergoing in Québec. We also present the results of some enquieries done with various samples of Québec's elementary teachers. We analyse various concepts or constructs (subject matter; discipline; basic or secondary subject matters; knowledge) following their impact on the epistemological foundations of the curriculum or the teaching processes. We finish our analysis by questionning the coherence between a constructivist discourse and the basic concepts who forms the ministry's discourse foundations that relates directly to a neobehaviourist stance.

1. Les auteurs sont membres du Groupe de recherche sur l'interdisciplinarité dans la formation à l'enseignement (GRIFE) ainsi que du Centre interuniversitaire de recherche sur la formation et la profession enseignante (CREFPE interuniversitaire). 


\section{RESUMEN}

\section{Curriculum y coca-cola : ¿una nueva presentación cambia el sabor? Los conceptos de materia, asignatura, saber y conocimiento en el contexto de la reforma del curriculum en Quebec}

François LAROSE

Universidad de Sherbrooke, Québec, Canadá

Vincent GRENON

Universidad de Sherbrooke, Québec, Canadá

Sébastien RATTÉ

Universidad de Sherbrooke, Québec, Canadá

Mary PEARSON

Universidad de Sherbrooke, Québec, Canadá

En este artículo realizamos un análisis de contenido comparativo del discurso de algunos de los actores principales de la reforma curricular et de profesionales de la enseñanza primaria de Quebec. Los conceptos estudiados, materia, asignatura, materia de base o secundaria y saber, fueron analizados tomando en consideración el impacto que tienen sobre las bases epistemológicas del curriculum y de la enseñanza. Terminamos el artículo cuestionando la pertinencia del recurso a un discurso que se concibe como constructivista siempre y cuando los conceptos sobre los que se apoya el discuro sobre la educacion se definan a partir de una epistemología realista cuya expresión curricular no puede ser que de tipo neobehaviorista.

\section{Introduction}

Dans son rapport présenté à la ministre de l'Éducation du Québec, le Groupe de travail sur la réforme du curriculum (Gouvernement du Québec, 1997a) proclamait " haut et fort » l'inutilité ou la non-pertinence de sept matières scolaires. Prenant prétexte des déclarations faites dans le cadre des États généraux de l'éducation (Gouvernement du Québec, 1996a) par différents corps constitués ou organismes représentant le monde de l'enseignement, les élèves ou la communauté, le Groupe de travail préconisait la suppression de ces matières scolaires dans le cadre d'une refonte en profondeur de la «grille-matières ». Le rapport du Groupe de travail sur la réforme du curriculum se conformait ainsi au mandat accordé par la ministre dans le cadre du plan d'action pour la réforme de l'éducation selon lequel, désormais, l'école devait enseigner les "matières essentielles» (Gouvernement du Québec, 1996b). En conséquence, le ministère de l'Éducation consacrait la pondération différenciée des matières scolaires, des savoirs auxquels renvoient ces matières et du 
champ d'appartenance de ces derniers: compétences "transversales», contenus constituant l'essence d'une "culture générale» ou encore savoirs instrumentaux indispensables (Gouvernement du Québec, 1997b).

D'emblée, en posant la question de «l'essentialité d'une matière » le Groupe de travail, puis le Ministère posent celle, plus complexe, du rapport au savoir qu'entretient l'enseignant ou l'élève ainsi que celle portant sur la nature sociale du savoir et sur le fondement relatif, socialement déterminé, de sa pondération. Ce faisant, tant le rapport au savoir que la conception privilégiée de ce que sont et de ce que représentent les matières scolaires s'avèrent déterminants au regard des orientations que l'État donnera à la formation initiale ou continue à la profession enseignante. Ainsi retrouve-t-on, dans les documents d'orientation de la formation à la profession enseignante publiés récemment, mention d'un certain nombre de concepts qualifiant de façon identique cette formation et les finalités du curriculum d'enseignement au primaire et au secondaire. Parmi ces concepts, mentionnons la construction des "compétences transversales" ainsi qu'une "solide culture générale». La formation initiale à la profession enseignante doit, de plus, viser la maîtrise des disciplines et des programmes d'études qui y sont rattachés ainsi que la construction de compétences de type techno-instrumental: « des compétences dans les domaines de la didactique, de la gestion de classe et de l'évaluation" (Gouvernement du Québec, 1997c, 1999).

Dans cet article, nous identifierons dans un premier temps le sens que les concepts de discipline et de matière scolaire adoptent selon la terminologie en usage en sciences de l'éducation. Nous examinerons ensuite le traitement qui en est fait dans le discours des divers intervenants gouvernementaux de l'actuelle réforme du curriculum, puis chez les enseignantes et les enseignants du primaire au Québec. À cet effet, nous utiliserons des données recueillies dans le cadre de deux recherches successives $^{2}$. Dans un deuxième temps, nous identifierons le rapport que les divers intervenants concernés par la réforme du curriculum entretiennent au regard de ce qu'est le savoir et ce qui définit sa construction ou son appropriation. Nous terminerons en soulignant la cohérence existant chez l'ensemble des décideurs et des intervenants du monde de l'enseignement primaire au regard des divers concepts analysés et en mettant en lumière les conséquences de cette cohésion sur le plan des orientations épistémologiques qui fondent l'enseignement primaire au Québec.

2. Il s'agit des recherches suivantes: Les représentations des titulaires de l'enseignement primaire québécois au regard de l'interdisciplinarité pédagogique et de son actualisation pédagogique. Chercheur principal : Yves Lenoir; cochercheur : François Larose (FCAR, 1992-1995); Compétences didactiques et formation didactique des enseignantes et des enseignants du primaire. Chercheur principal : Yves Lenoir; cochercheurs: François Larose et Carlo Spallanzani (CRSH 1995-1998). 


\section{Les concepts de discipline et de matière scolaire en perspective}

Le fait qu'une matière soit jugée « essentielle » (de base) ou secondaire renvoie à une pondération, généralement sociale, de son utilité et, partant, de sa pertinence au sein du curriculum scolaire. Cela étant, il y a jugement quant à la pertinence ainsi qu'à l'utilité sociale ou individuelle des savoirs propres à une ou à des disciplines scientifiques auxquelles cette matière scolaire renvoie (Bernstein, 1971; Goodson, 1987, 1997). Le concept de matière, qu'elle soit de base ou secondaire, renvoie à celui, fort controversé, de discipline scolaire. Il s'agit là d'un construit dont l'existence ne se justifie qu'en nuance, en complémentarité ou en opposition à celui de discipline scientifique.

Le concept de matière, tout comme celui de discipline d'ailleurs, renvoie à des univers plus ou moins circonscrits de connaissances ou de savoirs homologués dans certains cas, non homologués dans d'autres. La notion de critère de reconnaissance sociale s'applique donc encore une fois ici. Le savoir homologué, par définition, n'est pas un savoir de sens commun. La représentation sociale du champ que recouvre ce savoir homologué et des caractéristiques requises par l'apprenant ainsi que du processus d'apprentissage permettant d'y avoir accès, pour sa part, demeure du domaine du sens commun. Il s'agit là de la définition même de la représentation sociale et de ce qui la différencie de la représentation individuelle, cognitive, d'un objet ou encore de sa représentation socialisée (Larose, Audette et Roy, 1997a; Larose et Lenoir, 1997; Larose, Lenoir et Karsenti (à paraître); Moliner, 1995; Moscovici, 1984, 1988).

Jusqu'à quel point y a-t-il correspondance entre la définition sociale de ce que sont une discipline (scientifique), une matière scolaire, une matière essentielle ou secondaire, d'une part et la définition socialisée, de sens commun, de ce que représentent les mêmes construits, d'autre part? Pour y répondre, nous devrons examiner dans un premier temps les définitions formelles des divers concepts à l'étude. Dans un deuxième temps, nous confronterons ces définitions avec celles, moins formelles, qui se retrouvent plus ou moins clairement, souvent en filigrane, dans le discours gouvernemental récent. Enfin, nous confronterons ces définitions avec celles, de sens commun, qui structurent leur représentation au sein de deux entités particulières : les enseignantes et les enseignants québécois au primaire et les futurs enseignants et enseignantes; les étudiantes et les étudiants du baccalauréat en enseignement au préscolaire et au primaire (BEPP) de l'Université de Sherbrooke.

\section{Les concepts à l'étude selon diverses catégories d'intervenants}

\section{Les concepts de matière et de discipline selon le vocabulaire en usage en sciences de l'éducation}

Legendre (1993) distingue une définition « didactique » et une définition « scientifique » ou épistémologique du construit de discipline. Sur un plan didactique, la discipline correspond à « une branche du savoir pouvant faire l'objet d'un enseignement » 
(p. 138). Dans une perspective plus épistémologique, la discipline correspond à un « domaine structuré du savoir qui possède un objet d'études propre, un schème conceptuel, un vocabulaire spécialisé, ainsi qu'un ensemble de postulats, de concepts, de phénomènes particuliers, de méthodes et de lois» (Ibid.).

Si la discipline peut faire l'objet d'un enseignement, il importe alors de définir le concept de matière. Cette dernière se définit en tant que "partie d'une discipline, circonscrite dans un programme d'études, faisant l'objet d'un enseignement scolaire » (Ibid., p. 819). Bien que ne distinguant pas la matière de base de la matière secondaire, Legendre (1993) fournit quand même quelques indications au regard de ce que recouvre le concept de matière de base. Il s'agit d'une «matière qui comporte un ensemble de notions indispensables à l'apprentissage de connaissances ultérieures " (p. 820). Cette définition se rapproche assez de celle de De Landsheere (1979). Ce dernier entend par matières de base les matières outils, c'est-à-dire les «connaissances qui sont l'instrument des autres acquisitions, par exemple: la lecture, l'écriture, le calcul, l'habileté à consulter les ouvrages de référence, les connaissances informatiques, etc.» (p. 170). De façon nuancée et se référant de manière informelle au concept de curriculum, Legendre (1993) définit la matière "principale» en tant que matière "dont l'importance est prépondérante dans un programme d'études " (p. 821). Il est intéressant de constater que la définition que De Landsheere fait des matières de base est presque identique à celle que fait le Groupe de travail sur la réforme du curriculum, huit ans plus tard, au regard des compétences «transversales » d'ordre intellectuel et méthodologique (Gouvernement du Québec, 1997a).

\section{Les concepts à l'étude selon le discours gouvernemental}

Dans divers documents produits par le ministère de l'Éducation du Québec (MEQ), les concepts de discipline et de matière sont utilisés de façon indistincte, les deux termes étant souvent présents de façon concomitante, comme si leur alternance avait surtout une finalité stylistique. Recourir au terme discipline avant ou après celui de matière évite les redondances. Par ailleurs, nulle part dans les documents du Ministère ne trouve-t-on de définition particulière, distincte, de ce qu'est une discipline ou une matière scolaire. Les extraits suivants, tirés de l'énoncé de politique éducative, suffiront à illustrer notre propos.

Premièrement, une meilleure place sera réservée aux matières plus «naturellement» porteuses de culture, telles que les langues, les arts et l'histoire. Deuxièmement, on favorisera une approche culturelle pour enseigner ces matières. [...] Ainsi en est-il des productions culturelles rattachées à toutes les disciplines. Troisièmement, pour que cette perspective ne soit pas laissée à la seule initiative personnelle des enseignants et enseignantes, la révision des programmes d'études prévoira explicitement l'intégration de la dimension culturelle dans les disciplines (Gouvernement du Québec, 1997b, p. 13).

Les objectifs doivent être clairs et les exigences, les progressions d'une classe ou d'un cycle à un autre et les liens avec les autres disciplines 
doivent être explicites. Par ailleurs, des séquences seront établies pour toutes les matières afin d'indiquer la progression des apprentissages [...] (Gouvernement du Québec, 1997b, p. 14)

L'énoncé de politique guidant l'actuelle réforme curriculaire se fondant sur le résultat des travaux du Groupe de travail sur le curriculum (GTC), nous avons voulu vérifier si le discours de ce dernier se voulait plus nuancé en la matière [sic]. D’une façon générale, le rapport du Groupe de travail semble utiliser de façon tout aussi indistincte, quasi synonymique, les deux concepts. Les fragments suivants en feront foi :

La détermination de ces contenus revêt des formes différentes. Elle peut indiquer les grands champs des savoirs, les raisons qui justifient ces choix et les orientations qui doivent influencer l'enseignement des matières et des disciplines qui interviennent dans ces champs (Gouvernement du Québec, 1997a, p. 15).

Nous recommandons que certaines matières apparaissant présentement à la grille-matières du secondaire comme cours obligatoires n'en fassent plus partie comme disciplines autonomes, mais que leur contenu soit intégré dans d'autres matières ou que des activités les remplacent ou, enfin, que certains éléments ne fassent plus partie du curriculum d'études (Gouvernement du Québec, 1997a, p. 57).

Le Conseil supérieur de l'éducation (CSE) représente le troisième intervenant institutionnel à s'être prononcé à la fois sur le projet de refonte du curriculum et, partant, sur le rôle, l'avenir et l'agencement des « disciplines » et des « matières scolaires ". Plus précisément, dans deux documents successifs, le Conseil se prononce, tout d'abord sur le contenu et sur certaines faiblesses conceptuelles propres au rapport du Groupe de travail sur le curriculum, puis sur ce qui lui semble être les faiblesses et limites de la mise en œuvre actuelle de la réforme curriculaire (Conseil supérieur de l'éducation, 1998, 1999). Le recours au concept de matière ou de discipline ne semble guère plus nuancé ici que dans le discours ministériel ou dans celui du Groupe de travail.

Un curriculum bien équilibré et riche n'est pas un facteur d'échec plus grand qu'un curriculum étroit ressassant un petit nombre de matières « de base». Un curriculum hautement sensible à toutes les dimensions constitutives de la culture actuelle, y compris sa dimension pratique et technique, un curriculum hautement pertinent dans la sélection qu'il opère de chances d'apprendre à partir de la culture actuelle, est aussi mobilisant et plus universellement enrichissant qu'un curriculum censé favoriser l'excellence de quelques-uns par une hiérarchisation exagérée des disciplines (Conseil supérieur de l'éducation, 1999, p. 23).

En fait, lorsqu'il s'adresse à la production du Groupe de travail, production au sein de laquelle on renvoie plus généralement au concept de matière, simplement 
par effet de pratique (on y traite de la grille-matières), le Conseil utilise plus systématiquement le terme de matière que celui de discipline. Par contre, lorsqu'il procède à l'analyse de la mise en œuvre de la réforme curriculaire et, partant, qu'il dirige sa critique de façon plus ciblée sur l'énoncé de politique en vigueur et sur les documents qui en découlent, alors le Conseil utilise de façon plus systématique le terme de discipline. Tout se passe comme si, à concepts équivalents, voire indistincts, le discours utilise de façon plus ou moins importante, quantitativement, l'un ou l'autre d'entre eux tout en se moulant à la terminologie privilégiée par les auteurs dont les productions font l'objet d'une analyse critique.

De façon à confirmer ce qui précède, nous avons procédé à une analyse lexicométrique d'un corpus documentaire constitué de l'ensemble des paragraphes des quatre documents susmentionnés pour autant que ces derniers intègrent la mention du concept de matière ou de discipline. L'objet principal de cet article n'étant pas d'ordre méthodologique, mentionnons simplement que les énoncés composant le fichier numérisé à partir de l'ensemble des fragments retenus ont été traités sur la base d'une analyse factorielle des correspondances réalisée sur matrices de fréquences éparses à l'aide du logiciel Lexico ${ }^{3}$. L'analyse réalisée présente cependant une particularité. La matrice de fréquences à partir de laquelle le plan factoriel est produit en est une qui retient comme unité d'analyse le segment répété, donc toute structure lexicale composée de deux mots ou plus, présente au moins deux fois consécutives en forme identique dans le discours d'un sujet.

Le lecteur qui voudrait se familiariser avec les techniques d'analyse statistique des données textuelles se référera à Lebart et Salem (1994) ainsi qu'à Lebart, Salem et Berry (1997). Il trouvera aussi des exemples d'application de la statistique textuelle à l'analyse du discours en éducation en consultant Larose, Audette et Roy (1997b), Larose, Jonnaert et Lenoir (1996a) et Larose, Lavallée et Demers (1996b).

Une rapide consultation du tableau 1 permettra au lecteur de constater que le discours gouvernemental et paragouvernemental est plutôt faiblement polarisé. Le premier facteur (axe horizontal) se distribue d'une référence aux activités d'enseignement ainsi qu'aux matières scolaires (gauche) vers une préoccupation plus marquée au regard des finalités générales de l'éducation (droite). Le second facteur (axe vertical) varie entre la référence aux contenus qui définissent la «culture générale", les savoirs pondérés selon l'importance des matières scolaires (bas) et l'identification des matières "contenus » dont la finalité principale est de permettre «l'intégration des savoirs» (haut). Le fait que les deux premiers axes expliquent à eux seuls la totalité de la variance s'avère un phénomène relativement rare en statistique textuelle et reflète l'homogénéité, voire la répétitivité des éléments discursifs. Nous avons là un indice de faible variation des concepts de référence auxquels recourent les auteurs.

Certains termes caractérisent plus particulièrement le discours de l'un ou l'autre des organismes. Ainsi, le concept de matière est plus particulièrement fréquent dans

3. Les versions 1 et 2 du logiciel Lexico que les chercheurs du GRIFE utilisent ont aimablement été fournies par leur auteur, le professeur André Salem de I'Université de la Sorbonne Nouvelle à Paris. 
le discours partagé par le Ministère ainsi que par le Groupe de travail alors que celui de discipline, situé à droite du second axe factoriel (F2) dans le troisième quadrant, semble être plutôt l'apanage du Conseil. Néanmoins, seul le terme discipline représente une «forme banale» du discours, donc un élément textuel dont l'utilisation n'est pas particulièrement discriminante en fonction de l'origine des fragments. Pour sa part, le terme matière représente une spécificité négative, c'est-à-dire un élément discursif proportionnellement sous-représenté dans le discours du Conseil.

Tableau 1. AFC des segments répétés du discours du MEQ, du CSE ainsi que du GIC au regard de la matière scolaire ou de la discipline (scolaire)

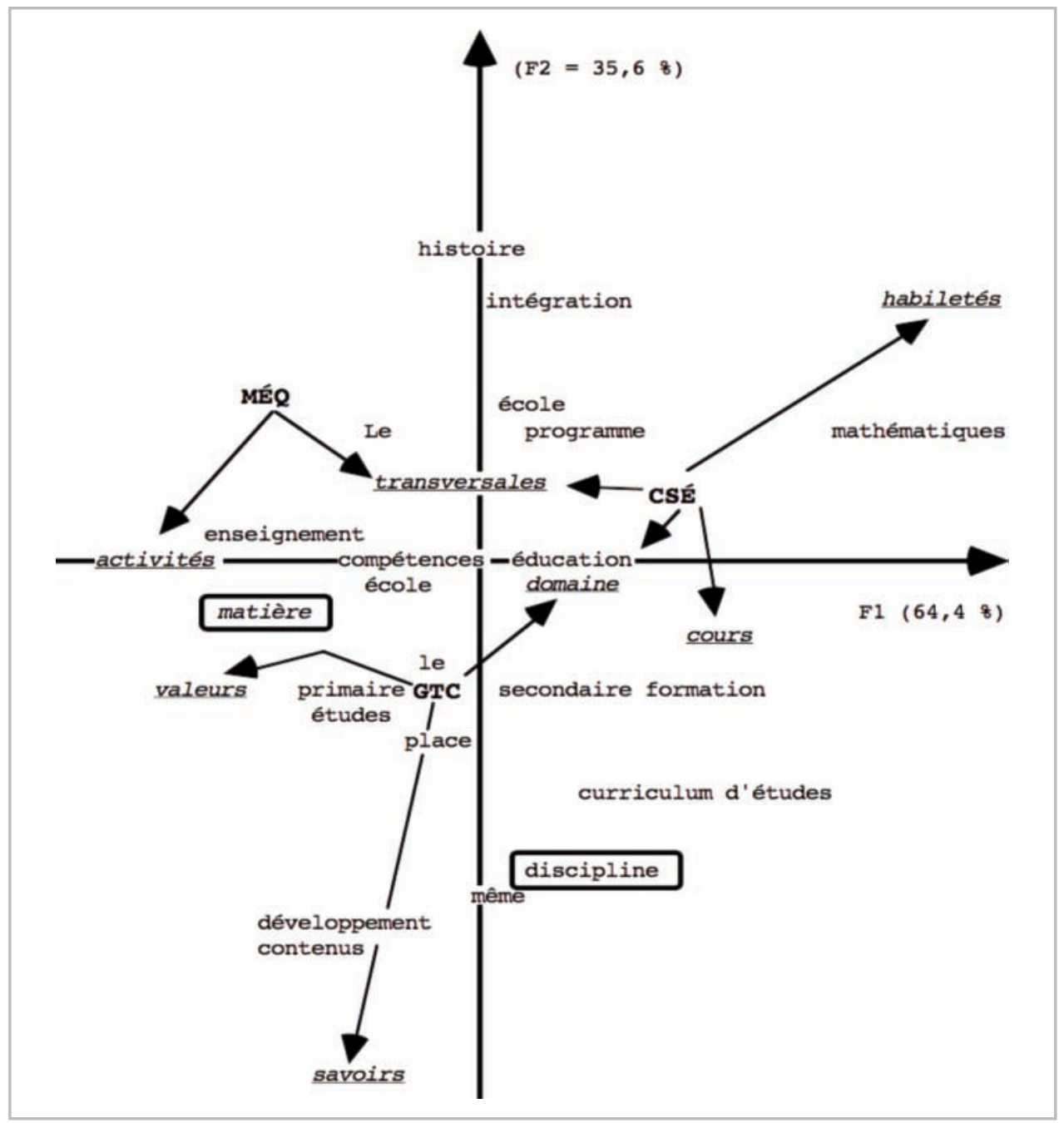

F1: Axe horizontal : référence aux activités d'enseignement / référence aux finalités de l'éducation. F2: Axe vertical : référence aux contenus / référence aux matières permettant l'intégration des contenus des matières « outils". 
Dans le tableau 1, les particularités du discours, en ce que les éléments discursifs sont sur-représentés chez l'un ou l'autre des trois organismes, sont illustrées par les mots à la fois soulignés et en italique. La référence aux habiletés est donc une spécificité du discours du CSE alors que l'intégration du concept de «savoir» au singulier caractérise de façon exclusive le discours du GTC. Par contre, la référence à la "transversalité » caractérise à la fois le discours du MEQ et du CSE, alors que ce concept est sous-représenté dans le discours du GTC.

\section{Les concepts à l'étude selon les futurs professionnels}

En février et en mars 1996, nous demandions à un échantillon de convenance formé d'étudiantes et d'étudiants des trois cohortes du programme de baccalauréat au préscolaire et au primaire de la Faculté d'éducation de l'Université de Sherbrooke de produire un ensemble de définitions de divers concepts. Parmi ceux-ci, les concepts de matière scolaire et de discipline. L'échantillon réunissait des étudiantes et des étudiants intégrés à la fois dans le cadre de l'ancien et du nouveau curriculum de BEPP (tableau 2).

Tableau 2. Structure échantillonnale (étudiantes et étudiants, CRSH 1995-1998; phase 1)

\begin{tabular}{|l|c|c|c|}
\hline Cohorte & N & \% Échantillon & \% Population \\
\hline BEPP, première année (nouveau programme) & 41 & 62 & 70 \\
\hline BEPP, deuxième année (ancien programme) & 20 & 30 & 40 \\
\hline BEPP, troisième année (ancien programme) & 05 & 08 & 17 \\
\hline
\end{tabular}

Nous avons procédé à l'analyse lexicométrique des définitions produites, tout d'abord en utilisant les formes (mots), puis les segments répétés (éléments de définition ou structures conceptuelles stables). En ce qui concerne la production d'une définition du concept de matière scolaire, seule l'AFC réalisée sur la matrice des mots permet de trouver un élément stable, commun à la majorité des définitions fournies. Cet élément correspond au "sujet " (thème d'un enseignement) qui se trouve associé chez les répondantes et les répondants de première année au concept de matière scolaire $\left(\mathrm{X}^{2}=2,54(41,123), p<0,006\right)^{4}$.

L'AFC réalisée sur la matrice des segments répétés ne permet pas de trouver de structure de définition stable, commune à la majorité des répondantes et des répondants, peu importe la cohorte (tableau 3). Néanmoins, bien que non significatif, le calcul des distances au chi carré les plus stables, pour chaque cohorte, permet de

4. $\mathrm{X}^{2}=$ Distance au chi carré. 
constater une évolution depuis une définition centrée sur les dimensions disciplinaires (première année) vers une centration curriculaire ou pédagogique. Cette dernière (énoncés caractéristiques des deuxième et troisième années) définit la matière scolaire en fonction de la division séquentielle, temporelle, des contenus qui font l'objet d'une période d'enseignement déterminée.

Tableau 3. AFC des définitions du concept de matière scolaire (énoncés les plus caractéristiques pour chacune des cohortes)

\begin{tabular}{|l|l|c|}
\hline Cohorte & Énoncé caractéristique & $\begin{array}{c}\text { Distance au Chi carré } \\
\text { ( } \boldsymbol{p} \text { atteint })\end{array}$ \\
\hline Première année & Contenu d'une certaine discipline & 0,370 \\
\hline Deuxième année & Différents sujets obligatoires se divisant en plusieurs points & 0,249 \\
\hline Troisième année & Le sujet d'étude & 0,287 \\
\hline
\end{tabular}

Par contraste, les étudiantes et les étudiants des trois cohortes produisent des définitions cohérentes et stables du concept de discipline. De la première à la troisième année, ces définitions évoluent d'une centration sur les contenus propres à l'univers scolaire vers une définition plus large, associant discipline et ensemble cohérent de connaissances ou de concepts, plus ou moins contextualisés (tableau 4).

Tableau 4. AFC des définitions du concept de discipline (énoncés les plus caractéristiques pour chacune des cohortes)

\begin{tabular}{|l|l|c|}
\hline Cohorte & Énoncé caractéristique & $\begin{array}{c}\text { Distance au Chi carré } \\
(\boldsymbol{p} \text { atteint })\end{array}$ \\
\hline Première année & Ensemble des connaissances d'une matière & 0,0001 \\
\hline Deuxième année & Ensemble des connaissances d'un domaine & 0,0001 \\
\hline Troisième année & Ensemble de contenus notionnels & 0,0001 \\
\hline
\end{tabular}

Dans le cadre de cette étude, nous ne disposions pas formellement d'information au regard de ce qui distingue une matière principale d'une matière secondaire. Néanmoins, l'analyse des résultats d'une tâche de classification hiérarchique des matières scolaires réalisée par la majeure partie des étudiantes et des étudiants du BEPP durant le semestre d'hiver 1997 permet de constater que plus de $73 \%$ des répondantes et des répondants $(\mathrm{N}=242)$ identifiaient de façon concomitante le 
français en tant que matière la plus importante et classait la mathématique au second rang. Une brève consultation du plan factoriel résultant de l'analyse factorielle d'un tableau de distances ${ }^{5}$ (MDS) des classements hiérarchiques permet de constater une division en blocs homogènes des matières scolaires (tableau 5).

L'axe horizontal reflète l'importance accordée à ces dernières, cette importance se distribuant de gauche (moindre importance) à droite (plus grande importance) La polarisation de l'axe démontre clairement la primauté du français et des mathématiques ainsi que le caractère marginal de l'enseignement religieux, de l'enseignement moral, de la danse, de la musique et de l'art dramatique.

\section{Tableau 5. MDS : Étudiantes et étudiants; classification hiérarchique des matières scolaires (CRSH 1995-1998; phase 2)}

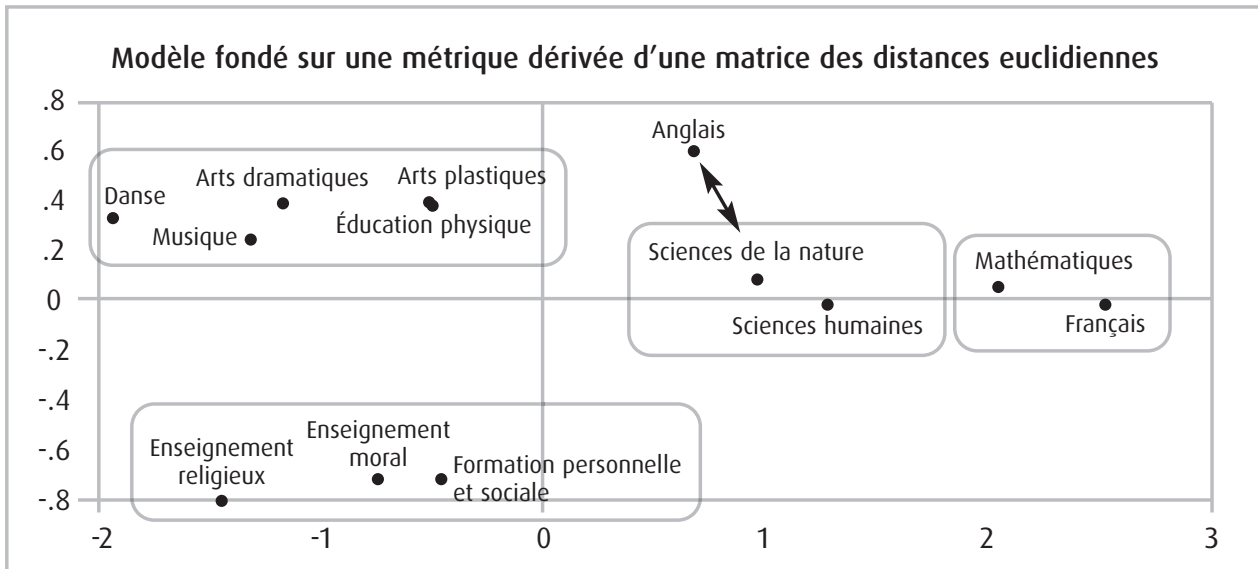

Axe horizontal : Gauche : matières classées moins importantes / Droite : matières classées plus importantes.

Axe vertical : Bas : Plus grande homogénéité des fréquences / Haut : Moindre homogénéité des fréquences.

La partition de l'ordre des matières reflète de façon fidèle celle qui fut constatée par divers chercheurs et organismes dans le cadre de cinq enquêtes réalisées auprès de praticiennes et de praticiens chevronnés entre 1981 et 1997 (Lenoir et Larose, 1999; Lenoir, Larose et Grenon (à paraître)). Les auteurs de ces diverses recherches constatent que le classement effectué par les enseignantes et les enseignants au regard de l'importance des matières scolaires correspond à une identification stable du français et des mathématiques en tant que "matière de base» et des autres matières en tant que «matières secondaires » (CSE, 1982; Laforest, 1989; Lenoir, 1991; Larose et Lenoir, 1998).

5. Traduction française de Multidimensional Scaling (MDS) proposée par Lebart, Morineau et Piron (1995). Pour se familiariser avec les divers modèles d'analyse multidimensionnelle, le lecteur pourra se référer à Mardia, Kent et Bibby (1979). 


\section{Les concepts à l'étude selon le discours des professionnels chevronnés}

Dans le cadre de deux recherches successives, nous avons recueilli des données sur les divers concepts à l'étude auprès d'un échantillon de 242 enseignantes et enseignants chevronnés intervenant à l'ordre primaire dans l'ensemble du Québec à l'exception du territoire de l'île de Montréal. Lors de la première recherche, les intervenantes et les intervenants étaient conviés à compléter une tâche d'association identifiant une structure de synonymes ou d'expressions jugées équivalentes notamment aux concepts de matière et de discipline.

Si $18,2 \%$ des répondantes et des répondants identifiaient celui de discipline en tant que synonyme du concept de matière, près de $38 \%$ d'entre elles et d'entre eux ont identifié le concept de matière en tant qu'équivalent de celui de discipline. Le concept de matière semble relativement stable sur le plan du champ sémantique. En effet, près de $60 \%$ des répondants ont identifié ce concept aux objets cognitifs d'un enseignement. Inversement, le concept de discipline semble relativement polysémique, $41,7 \%$ des répondantes et des répondants l'identifiant à un objet d'enseignement contextualisé (matière ou sujet) alors que près de $23 \%$ d'entre eux associaient le concept de discipline aux objets de la gestion de classe. Le lecteur trouvera une classification des principaux concepts jugés équivalents au Tableau 6.

Tableau 6. Classification des principaux concepts équivalents à ceux de matière et de discipline

\begin{tabular}{|l|c|c|l|c|c|}
\hline \multicolumn{4}{|c|}{ Équivalent conceptuel } & \multicolumn{3}{c|}{ Équivalent conceptuel } \\
\hline Matière & $\mathrm{N}$ & $\%$ & Discipline & $\mathrm{N}$ & $\%$ \\
\hline Discipline & 31 & 18,2 & Matière & 64 & 37,6 \\
\hline Sujet d'étude & 30 & 17,7 & Sujet & 07 & 00,5 \\
\hline Contenu d'enseignement & 26 & 15,3 & Ordre & 17 & 01,0 \\
\hline Domaine d'étude & 14 & 08,2 & Règles/règlement & 22 & 12,9 \\
\hline Total & 101 & $\mathbf{5 9 , 4}$ & Total & 110 & 64,6 \\
\hline $\begin{array}{l}\text { Identification hors du champ } \\
\text { de la pratique d'enseignement }\end{array}$ & 26,4 & Matière + sujet & & 41,7 \\
\hline $\begin{array}{l}\text { Identification à l'intérieur du champ } \\
\text { de la pratique d'enseignement }\end{array}$ & 33,0 & Comportement & & 22,9 \\
\hline
\end{tabular}

Nous ne disposions pas d'information directe (définition conceptuelle) de ce qui spécifie ou distingue la matière scolaire de la discipline dans le cadre de la recherche Fonds pour les chercheurs et l'aide à la recherche (FCAR). Cependant, 180 enseignantes et enseignants nous ont fourni une définition de ce que sont une 
matière de base et une matière secondaire. L'analyse lexicométrique des réponses permet de constater que la définition que les enseignants donnent de chacune des deux notions s'avère à la fois très dense et unitaire. La représentation en est donc très homogène. L'analyse factorielle des correspondances des éléments de définition fournis permet d'identifier une structure commune de ce que sont une matière de base et une matière secondaire (tableau 7). Les matières de base seraient centrées sur le développement d'habiletés d'ordre cognitif, alors que les secondes viseraient essentiellement celui des dimensions affectives chez l'élève.

Tableau 7. AFC des définitions d'une matière de base et d'une matière secondaire

\begin{tabular}{|l|l|c|}
\hline $\begin{array}{l}\text { Type de } \\
\text { matière }\end{array}$ & Énoncé caractéristique & $\begin{array}{c}\text { Distance au Chi carré } \\
\text { ( } \boldsymbol{p} \text { atteint) }\end{array}$ \\
\hline De base & $\begin{array}{l}\text { Une matière essentielle à la formation d'un individu } \\
\text { et nécessaire au développement d'habiletés diverses. }\end{array}$ & 0,0003 \\
\hline Secondaire & $\begin{array}{l}\text { C'est une matière qui vient compléter le développement } \\
\text { intégral de l'enfant. Ces matières aident à son } \\
\text { épanouissement et à sa culture générale. }\end{array}$ & 0,0018 \\
\hline
\end{tabular}

$\mathrm{Au}$ cours des recherches subventionnées successivement par le FCAR et le Conseil de recherches en sciences humaines du Canada (CRSH) de 1992 à 1998, les enseignantes et les enseignants devaient accomplir une tâche de classification des matières selon leur importance ainsi que selon leur statut de matière de base ou de matière secondaire. Nous avons intégré les données produites par la réalisation de ces tâches dans le cadre de deux analyses factorielles de tableaux de distances réalisées de façon concomitante ${ }^{6}$ (tableaux 8 et 9).

Comme le lecteur pourra le constater, dans les deux recherches les intervenantes et les intervenants identifient clairement les matières essentielles ou « de base» au français ainsi qu'aux mathématiques. Dans les deux cas aussi leur classement hiérarchique réserve une place privilégiée à deux matières scolaires considérées, certes, secondaires, mais dont l'importance relative se détache nettement de celle des autres : les sciences humaines ainsi que les sciences de la nature. Ces résultats sont conformes à ceux obtenus par divers organismes et chercheurs de 1981 à 1997, tel que nous l'avons mentionné antérieurement. Dans l'ensemble de ces recherches, le français et les mathématiques occupent toujours les deux premières places dans l'ordre d'importance des matières scolaires. Ce sont aussi toujours elles qui sont considérées en tant que matières essentielles ou "de base». Dans les recherches mentionnées comme dans celles dont nous faisons ici état, quatre matières constituent systématiquement un second bloc: les sciences humaines, les

6. Les deux modèles factoriels expliquent chacun plus de $98 \%$ de la variance totale. 
sciences de la nature, l'anglais et l'éducation physique. Viennent ensuite les autres programmes, dont les arts, qui ferment la marche.

Tableau 8. MDS : Enseignantes et enseignants; classification hiérarchique des matières scolaires et identification des matières de base (CRSH 1995-1998; phase 2)

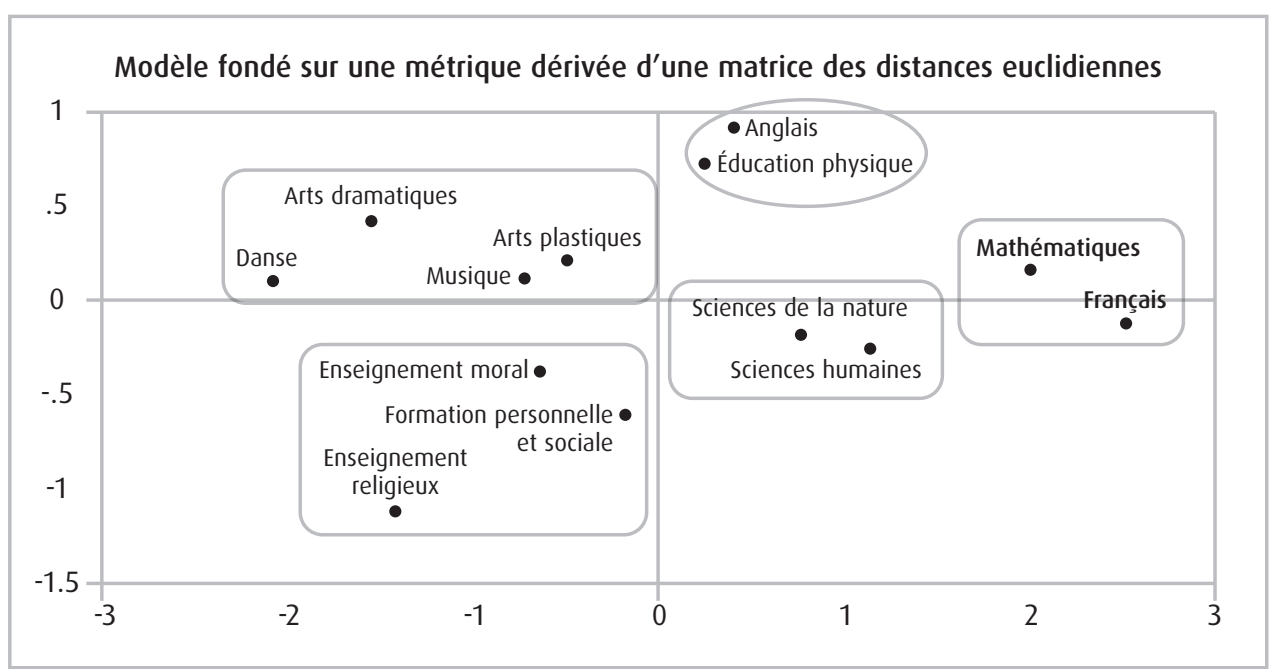

Gras: matières de base; fonte normale : matières secondaires.

Axe horizontal : Gauche : matières classées moins importantes / Droite: matières classées plus importantes.

Axe vertical : Bas: Plus grande homogénéité des fréquences / Haut : Moindre homogénéité des fréquences. 
Tableau 9. MDS : Enseignantes et enseignants; classification hiérarchique des matières scolaires et identification des matières de base (FCAR 1992-1995)

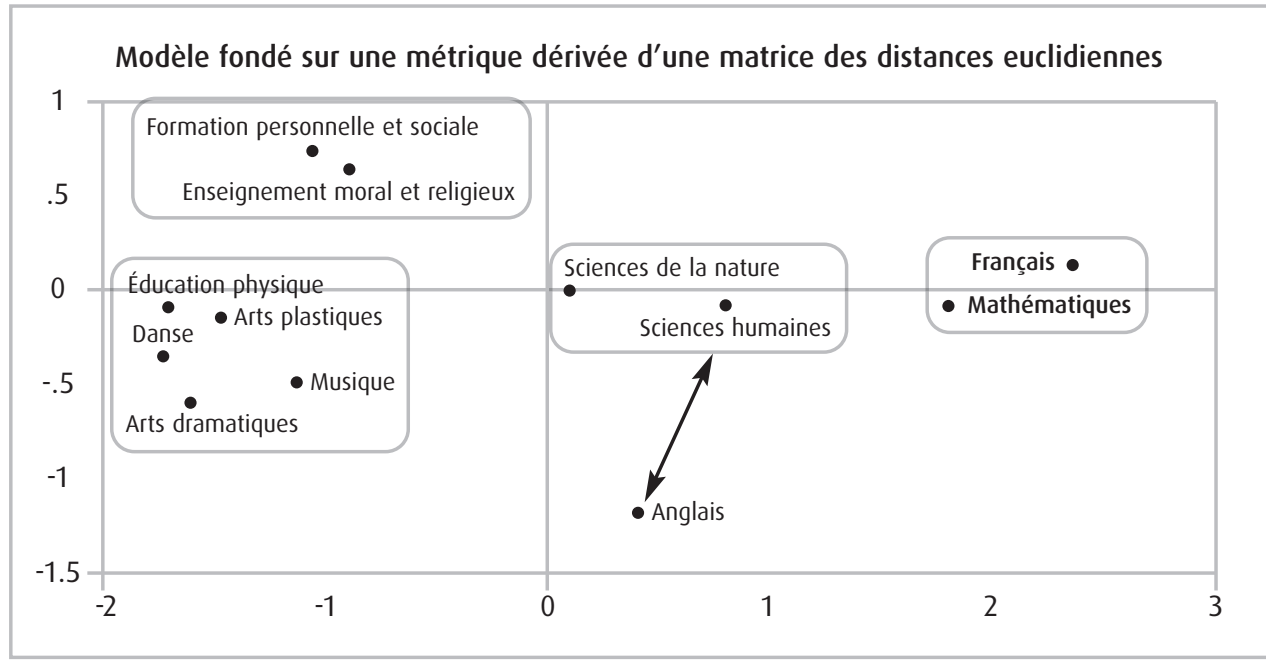

Gras : matières de base; fonte normale : matières secondaires.

Axe horizontal : Gauche : matières classées moins importantes / Droite : matières classées plus importantes.

Axe vertical : Bas: Plus grande homogénéité des fréquences / Haut : Moindre homogénéité des fréquences.

Dans l'ensemble des recherches mentionnées, les arts, saisis dans leur ensemble ou selon les différents volets qui les composent, se retrouvent, sauf une exception (Laforest, 1989), aux dernières places dans la hiérarchisation. Par ailleurs, le fait que les matières soient enseignées par la titulaire ou par une spécialiste ne semble pas avoir un impact au niveau de la classification. En fait, en tenant compte de la structuration des programmes d'études établie par Lenoir (1991), les enseignants allouent globalement, à l'exception de l'éducation physique, la première place aux programmes qui assurent l'expression de la réalité (français et mathématique) et la deuxième aux programmes qui permettent la construction de la réalité (sciences de la nature, sciences humaines). Les programmes qui établissent une relation à la réalité (ex: formation personnelle et sociale) viennent en troisième et les arts, qui assurent tout à la fois ces trois fonctions, mais sous un autre angle d'approche, en quatrième et dernière place. Par ailleurs, la partition des matières en deux blocs de première importance correspond aussi à la division entre matières "outils" (langue maternelle et mathématiques) et matières "contenus» (sciences humaines et sciences de la nature) qui fut mise en avant par Angers et Bouchard $(1984,1990)$ dans le cadre de la mise en œuvre des politiques du MEQ sur l'intégration des matières scolaires. 


\section{À l'origine de la matière scolaire ou de la discipline, le savoir}

\section{Savoir, processus d'accès au savoir et connaissance}

Si comme nous l'avons vu précédemment, tant le discours ministériel que celui du CSE ainsi que celui du GTC ne semble pas distinguer les concepts de matière scolaire et de discipline, quel rapport ces organismes entretiennent-ils avec le savoir? Cette question s'avère fondamentale, puisqu'elle met en jeu les fondements épistémologiques de l'ensemble de l'éducation ou de la formation scolaire. En effet, le savoir peut être conçu en tant qu'entité préexistante, externe à l'individu, une expression du réel per se ou en tant qu'expression du produit, absolu ou relatif, d'un rapport de construction, d'élaboration d'une représentation du réel par l'individu, un rapport au savoir distinct. Le savoir peut être conçu, de façon concomitante, comme produit de l'expression articulée d'une modélisation sociale du réel ou plutôt en tant que produit individuel des pratiques d'interaction directe et des pratiques sociales de chaque être humain, enfant ou adulte. Cela implique aussi une certaine conception du rapport au savoir qui, à son tour, détermine la structuration des processus d'enseignement-apprentissage donc, du curriculum (Larose et Lenoir, 1998).

\section{Savoir ou connaissance?}

Le concept de savoir tend souvent à être confondu ou, selon le cas, à être étroitement interrelié avec celui de connaissance (Dandurand et Ollivier, 1991). Pour ces auteurs :

la notion de connaissance a plus d'extension que celle de savoir : elle signifie toute appréhension symbolique de la réalité. Savoir, par ailleurs, est entendu dans le sens d'un processus plus restreint d'appréhension du réel renvoyant à la connaissance scientifique qui obéit à la rationalité scientifique et est soumise à des procédures définies de vérification; ou encore, la notion de savoir est employée dans la mesure où elle renvoie à des connaissances plus près des pratiques et devient à la limite, l'équivalent de savoir-faire (de skill [...]). Le savoir, enfin, aurait pour caractéristique de se présenter comme une pratique sociale réflexive (p. 4).

Ces deux auteurs se réfèrent à Beillerot $(1979,1989)$ pour qui le savoir est étroitement lié à sa mise en œuvre, à la pratique, « au savoir-faire parce qu'il n'existe réellement que par l'action qu'il permet» (p. 180). Dans ce sens, la connaissance désignerait l'ensemble de la production intellectuelle.

Ainsi, le savoir, qui ne peut se confondre avec la science, apparaît davantage lié à l'activité du sujet, à ses pratiques discursives. Mais Beillerot (Ibid.) en montre toute l'ambiguïté. Savoir et connaissance prennent des sens différents selon le système de référence auquel il est fait appel:

[...] si connaissance est entendue au sens de familiarité, intuition, sensibi-

lité, alors le savoir est plus intellectuel, plus conceptuel, et désigne un corps 
d'énoncés; et si la connaissance s'oppose à l'action, elle désigne toute la production intellectuelle. Quand les savoirs renvoient au savoir-faire et à l'empirie, la connaissance, au sens subjectif, resterait attachée à l'exercice de la pensée, à la représentation par l'idée de l'objet et du réel. D'un autre point de vue, si les savoirs d'un sujet ou d'une société peuvent se comprendre comme l'ensemble des savoirs stockés, les connaissances, elles, ne nommeraient que les savoirs spécifiques, ceux notamment appris par l'école ou l'étude. Il y a donc bien place pour un usage différent de savoir(s) et de connaissance(s) (Beillerot 1979, 1989, p. 180).

La dualité du concept de savoir se reflète clairement dans la façon dont Legendre (1993) par exemple, en structure la définition dans son dictionnaire. L'auteur respecte la dualité qualitative du concept, renvoyant à la fois à une caractéristique individuelle «avoir présent à l'esprit un ensemble d'informations, d'idées, de données, qui constituent des connaissances relatives à un domaine d'activités, à un objet, à une personne, etc.» (p. 1134) ou à un résultat de procès de construction collectif «Ensemble des connaissances systématisées, construit au cours de l'histoire, par des penseurs, des savants, etc. » (Ibid.). Dans ce contexte, le savoir peut être d'ordre global ou général, tel que précédemment défini ou, au contraire, spécifique ou disciplinaire, "[s] omme des connaissances spécifiques à une discipline » (Ibid.). Enfin, l'auteur y reconnaît la dimension de produit d'apprentissage pouvant avoir été réalisé en contexte formel ou informel «ensemble des connaissances approfondies acquises par un individu, grâce à l'étude et à l'expérience » (Ibid.).

Legendre (1993) distingue aussi entre savoir et connaissance, précisant que «[l]e savoir présuppose des acquisitions plus approfondies que la connaissance » (p. 1135). Cependant, lorsqu'il définit cette dernière, l'auteur y reconnaît la même dualité conceptuelle entre "processus" et "produit», de même qu'entre caractère général et caractère spécifique que lorsqu'il se réfère au savoir; «Faits, informations, notions, principes qu'on acquiert grâce à l'étude, à l'observation ou à l'expérience. [...] Totalité ou partie des données acquises à une époque, par une société ou par une personne. [...] Opération intellectuelle par laquelle un objet est rendu présent aux sens ou à l'esprit; fait ou manière de connaître; son résultat» (p. 240).

\section{Les concepts de savoir et de connaissance dans la littérature gouvernementale}

Dans son rapport final, le Groupe de travail sur le curriculum (Gouvernement du Québec, 1997a) n'offre aucune définition explicite de ce que représente le savoir ou la connaissance. Néanmoins, l'analyse des fréquences d'utilisation du concept de savoir présenté au singulier (le savoir) ou au pluriel (les savoirs), en tenant compte du contexte de référence, permet de constater que, si le recours au savoir en tant qu'entité générale (le savoir) est marginal dans le texte, l'utilisation d'un savoir pluriel, directement associé aux contenus descriptif (savoirs) ou procédural (savoirfaire), y est largement présente. Qu'on en juge. Sur l'ensemble des mentions du terme «savoir », le GTC ne renvoie que dans $4 \%$ des cas au concept de savoir en tant 
qu'entité globale, alors qu'il traite dans $89 \%$ de ses mentions des savoirs pluriels ou, si l'on préfère, du savoir en tant que somme circonscrite de connaissances descriptives identifiées aux disciplines ou aux matières scolaires. Enfin, le document ne renvoie que dans $5 \%$ des cas au concept de savoir dans sa dimension procédurale (savoir-faire). Le rapport proportionnel des références au concept de connaissance « singulière » ou, au contraire, «plurielles » est identique à la partition observée pour la notion de savoir. Dans $62 \%$ des cas, lorsqu'il utilise le terme de connaissance, le groupe de travail le fait de façon plurielle et fait directement référence aux univers de contenus, déclaratifs et procéduraux, propres aux matières scolaires. Le pourcentage résiduel des mentions ( $48 \%$ ) est essentiellement réparti entre l'utilisation du terme en tant qu'action de l'apprenant (la connaissance de quelque chose) et, secondairement, son utilisation en tant qu'univers de référence large équivalent au «savoir » en tant qu'entité globale.

L'énoncé de politique du Ministère (Gouvernement du Québec, 1997b) ne présente pas, non plus, de définition claire, directe, du concept de savoir. Dans $50 \%$ de cas, lorsqu'il mentionne le concept, le Ministère renvoie aux savoirs identifiés en tant que contenus (connaissances descriptives) et dans $25 \%$ aux dimensions procédurales (savoir-faire) associées aux matières scolaires. Lorsqu'il s'adresse à un savoir singulier ( $25 \%$ des mentions), le document renvoie en proportions égales à deux sens distincts. «Savoir» désigne alternativement une caractéristique individuelle de l'élève ou l'agrégat des connaissances humaines: «les différents domaines du savoir " (p. 23). Dans le premier cas, le Ministère fait référence à une capacité individuelle dans un sens identique à celui qu'il donne au «savoir-faire ", comme il le fait lorsqu'il traite de l'importance des apprentissages prescrits en mathématiques : «Il faut donc connaître et savoir pratiquer ces modes de calcul [...] il est essentiel de savoir lire et interpréter les données" (p. 17). Lorsqu'il renvoie au concept de connaissance, le Ministère le fait essentiellement au singulier. Ce faisant, il attribue au terme le sens du résultat d'un rapport individuel du sujet apprenant (l'élève) à un objet circonscrit. Il s'agit de la connaissance qu'un individu a, par exemple, de l'histoire de sa collectivité d'appartenance. Cette acception vaut pour la moitié des mentions. Les occurrences résiduelles renvoient au concept de connaissances plurielles ou, si l'on préfère, aux connaissances déclaratives et procédurales propres et particulières aux matières scolaires.

Enfin, ni dans son avis intitulé Pour un renouvellement prometteur des programmes à l'école (Conseil supérieur de l'éducation, 1998), ni dans son second document traitant de la réforme curriculaire Les enjeux majeurs des programmes d'études et des régimes pédagogiques (Conseil supérieur de l'éducation, 1999), le Conseil ne fournit de définition particulière des concepts à l'étude. Dans les deux documents analysés, la référence au savoir pluriel occupe plus de $50 \%$ des mentions faites du concept. Dans $25 \%$ des cas, on renvoie de façon particulière aux habiletés intellectuelles nécessaires pour opérer sur des objets de connaissance ou, si l'on préfère, aux connaissances procédurales (savoir-faire). Tout comme le Ministère, le Conseil, lorsqu'il fait référence à un savoir singulier, alterne entre un renvoi aux caractéristiques individuelles de l'élève résultant du processus d'apprentissage scolaire (50\% 
des mentions) et une relation à une entité plus large, plus globale. Néanmoins, dans ce dernier cas, contrairement au Ministère, le Conseil traite généralement des univers de connaissance propres aux disciplines ou aux matières scolaires. Cela étant, le Conseil ne renvoie pas, non plus, au concept de connaissance au singulier, en tant qu'équivalent du "savoir», mais bien plutôt en tant que contenus propres aux matières scolaires ou aux habiletés techno-instrumentales que l'individu, ou le futur citoyen, doit spécifiquement détenir dans le cadre de ce que prescrit le curriculum ou qu'il peut avoir acquises sur une base expérientielle.

\section{Les concepts de savoir et de connaissance chez les professionnels de l'enseignement}

Dans le cadre de la recherche FCAR (1992-1995), un sous-échantillon d'enseignantes et d'enseignants chevronnés venant essentiellement de Montérégie et de la grande région de Québec avaient accepté de participer à des entrevues de relance dont les questions portaient, notamment, sur leur conception du savoir. Dans le cadre de la recherche CRSH subséquente (1995-1998), une tâche identique était demandée à un échantillon initial d'enseignantes et d'enseignants de la région de Sherbrooke alors que les étudiantes et les étudiants étaient invités à nous fournir une définition personnelle de ce concept.

\section{Les concepts à l'étude selon les futurs professionnels}

Chez les sujets de l'échantillon étudiant, les concepts de connaissance et de savoir sont peu distincts (tableau 10). Pour eux, le savoir correspond à un univers de connaissances à caractère descriptif ou normatif, qui définissent le rapport entre un individu et un ensemble d'objets conceptuels plutôt flou en début de curriculum. Chez les sujets de deuxième et de troisième année, cependant, le savoir se définit en fonction d'un univers de référence ou d'un domaine de référence plus circonscrit. Il ne s'agit plus d'un savoir qualifiant l'individu au sens de l'érudit, mais bien plutôt la maîtrise de connaissances propres à un domaine (deuxième année) ou à un objet réel ou symbolique (troisième année).

Tableau 10. AFC des définitions du concept de savoir (CRSH 1995-1998; phase 1)

\begin{tabular}{|l|l|c|}
\hline Cohorte & Énoncé caractéristique & $\begin{array}{c}\text { Distance au Chi carré } \\
\text { ( } \boldsymbol{p} \text { atteint) }\end{array}$ \\
\hline Première année & Le savoir est une connaissance globale de plusieurs choses. & 0,0001 \\
\hline Deuxième année & C'est la connaissance acquise ou à acquérir dans un domaine. & 0,001 \\
\hline Troisième année & C'est la connaissance que l'on a de quelque chose. & 0,0001 \\
\hline
\end{tabular}


Chez les enseignantes et les enseignants, la représentation du savoir est plus délimitée (tableau 11). Il représente essentiellement une caractéristique individuelle, résultant d'un processus d'apprentissage formel ou informel. Il tend à correspondre à un cumul de connaissances portant sur des objets particuliers. Chez les enseignantes et les enseignants ayant participé à la première phase de la recherche CRSH (1995-1998), le savoir semble se définir de façon semblable à l'apprentissage et, notamment à l'apprentissage scolaire. La connaissance est le résultat inféré d'un processus. Il y a savoir, comme il y a eu apprentissage, lorsque l'élève ou l'individu est capable de réaliser une tâche qu'il n'était pas apte à faire antérieurement à l'exposition à une séquence de démonstration, d'enseignement, etc. Le savoir est donc une entité contextualisée. Les enseignantes et les enseignants nous parlent alternativement d'une caractéristique qualifiant l'individu (FCAR) ou de caractéristique qualifiant l'élève, de savoir scolaire (CRSH).

Tableau 11. AFC des définitions du concept de savoir (Enseignantes et enseignants : énoncés les plus caractéristiques)

\begin{tabular}{|l|l|c|}
\hline Cohorte & Énoncé caractéristique & $\begin{array}{c}\text { Distance au Chi carré } \\
\text { ( } \boldsymbol{p} \text { atteint) }\end{array}$ \\
\hline $\begin{array}{l}\text { FCAR } \\
1992-1995\end{array}$ & $\begin{array}{l}\text { Le savoir, c'est le résultat d'apprentissages et d'expériences. Le résultat des apprentissages, } \\
\text { quand j'exprime un savoir, c'est que j'ai vécu des expériences, j'ai appris, donc au bout du } \\
\text { compte je sais. C'est l'ensemble des connaissances d'un individu. }\end{array}$ & 0,03 \\
\hline $\begin{array}{l}\text { CRSH } \\
1995-1998\end{array}$ & $\begin{array}{l}\text { Ce sont les connaissances, les habiletés. C'est le résultat de l'apprentissage, un ensemble } \\
\text { de connaissances pour l'enfant. Des stratégies pour comment résoudre ses problèmes, se pré- } \\
\text { parer à la vie future parce que dans sa tête il faut qu'il soit organisé, il faut qu'il traite l'infor- } \\
\text { mation, il faut qu'il arrive à la conclusion. C'est tout le processus qui est en action. Le savoir, } \\
\text { moi je dirais, l'enfant qui résout un problème quand il arrive à une solution : il y a savoir. }\end{array}$ & 0,009 \\
\hline
\end{tabular}

D’une façon générale, nous pouvons considérer que tant le discours des intervenants gouvernementaux que celui des praticiennes et des praticiens, en formation ou en exercice, sont essentiellement centrés sur une définition plurielle du concept de «savoir». Cependant, si lorsqu'il fait référence marginalement au savoir au singulier, le discours officiel renvoie à une accumulation sociale de connaissances, celui des intervenants et des intervenantes définit essentiellement le résultat d'un processus d'apprentissage ou, si l'on préfère, une caractéristique de l'individu apprenant.

\section{Discussion}

Le vocabulaire en usage en sciences de l'éducation distingue, du moins sur le plan épistémologique, la discipline en tant que domaine structuré de savoir et la matière scolaire en tant que partie circonscrite de cette dernière, agencée de façon 
spécifique dans un programme d'études et devant faire l'objet d'un enseignement scolaire. Ce vocabulaire qualifie aussi la matière de base, non pas en tant que matière, donc partie spécifique de l'enseignement d'une discipline scientifique, mais bien, plutôt, en tant que matière véhiculant un ensemble de notions indispensables à l'apprentissage de connaissances ultérieures. Outre que cette qualification de la matière de base peut fort bien être considérée depuis l'angle d'approche qu'adoptent le rapport du Groupe de travail sur le curriculum ainsi que le Conseil supérieur de l'éducation lorsqu'ils traitent de «compétences transversales», la définition discriminante de la matière de base pourrait bien s'adresser à toutes les matières scolaires. En effet, peut-on identifier un programme d'enseignement qui ne présente pas son contenu de façon hiérarchisée? Existe-t-il un programme qui fasse l'économie d'une mise en relation des apprentissages ciblés avec certains préalables identifiés aux contenus explorés ou aux "compétences construites " lors d'une séquence d'études antérieure de la part des élèves?

Les organismes gouvernementaux dont nous avons analysé les textes, en l'occurrence le Groupe de travail sur le curriculum, le ministère de l'Éducation et le Conseil supérieur de l'éducation, pour leur part, ne distinguent pas entre les concepts de discipline ou de matière scolaire. Les deux entités semblent parfaitement synonymiques et leur usage en alternance indique plus un besoin d'allègement de la stylistique que l'existence d'une différenciation de niveau conceptuel. Par ailleurs, bien qu'ils soient peu nuancés sur le plan du discours lui-même, les organismes gouvernementaux distinguent entre matière de base et matière secondaire. Ils le font par la justification des matières scolaires jugées «superflues" sur le plan de la réorganisation de la grille-matières, sacrifiant en conséquence sept matières.

Chez les futurs enseignants et enseignants, on ne distingue pas de définition unitaire, stable, de la matière scolaire. Par contre, le concept de discipline se définit de façon restrictive par rapport aux connaissances d'une matière, d'un domaine ou encore en tant qu'ensemble cohérent de contenus notionnels. Chez les enseignantes et les enseignants chevronnés, les deux concepts sont peu distingués, la définition de l'un renvoyant au libellé de l'autre. Pour les praticiennes et les praticiens, la matière de base est celle qui s'avère essentielle à la formation de l'élève et nécessaire au développement d'habiletés diverses. La matière secondaire renvoie, pour sa part, au champ de la culture générale, personnelle, du sujet apprenant.

L'analyse des classifications hiérarchiques des matières, tant chez les étudiantes et les étudiants que chez les praticiennes et praticiens, permet d'identifier deux matières qui ressortent comme étant, de loin, les plus importantes. Il s'agit des mathématiques et du français. Deux matières scolaires « contenus » ressortent subséquemment. Il s'agit des sciences humaines et des sciences de la nature. L'ensemble des autres matières scolaires, à l'exception de l'anglais et de l'éducation physique, sont non seulement "fort secondaires", mais tellement peu valorisées qu' on peut se demander quelle est leur utilité dans le curriculum, selon les enseignants, si ce n'est de participer à la culture générale de l'enfant...

L'absence de distinction, qui semble généralisée chez les intervenants du monde de l'éducation, entre discipline et matière scolaire et, inversement, la stabilité de la 
distinction établie entre matières de base identifiées aux mathématiques et au français d'une part et les autres considérées en tant que matières secondaires, nous questionnent. Si le concept de matière est un équivalent direct de celui de discipline, il n'existe alors aucune spécificité de la discipline scientifique. Dans ce sens, la matière scolaire et la discipline ne font qu'un et, en utilisant comme référent conceptuel la définition de Legendre (1993), la matière scolaire n'est que la transposition du discours disciplinaire, scientifique, dans un contexte scolaire. Cette position, si elle s'avère correspondre à celle des organismes gouvernementaux ainsi qu'à celle des enseignantes et des enseignants, est lourde d'implications pour le sens qu'on donne au concept de «transposition». Celle-ci devient alors la réécriture des corpus de connaissances disciplinaires en des termes et selon un agencement séquentiel qui rendent ces derniers accessibles à l'apprenant selon ses caractéristiques développementales. Dans cette perspective, il n'y a pas de spécificité à la matière scolaire.

L'identité conceptuelle, liée à la stabilité de la définition des matières de base et à leur exclusivité en tant que matières liées à l'expression de la réalité, est aussi lourde d'implications quant aux fondements épistémologiques du savoir, de son statut et du rapport au savoir qu'exprime la relation enseignement-apprentissage. $\mathrm{Si}$, comme tend à le suggérer le résultat de l'analyse du discours des organismes gouvernementaux, pour ces derniers le savoir s'exprime essentiellement en tant qu'entité plurielle associée aux matières ou aux disciplines, le savoir est une donnée. Il représente l'organisation des connaissances construites dans le cadre des disciplines scientifiques et transposées de façon spécifique dans celui des matières scolaires. Dans une telle perspective, le savoir ne peut être qu'un donné extérieur à l'élève, une description de "la réalité» que l'enseignement doit lui permettre de découvrir soit par révélation, soit par un processus d'approximation guidée. De toute façon, peu importe l'angle d'approche, le savoir implique une dimension de normativité, les connaissances, déclaratives ou procédurales «à découvrir " possédant des propriétés fixes, décrites à jamais par la science, que l'élève peut maîtriser ou apprendre à utiliser de façon plus ou moins exacte, plus ou moins appropriée.

Si l'élève accède au savoir, peu importe la manière, c'est la correspondance entre la représentation qu'il en manifestera et le corpus de données que représente le savoir homologué, hiérarchisé, structuré dans le cadre des programmes et du matériel didactique qui en permettra la certification. Dans cette logique, le savoir équivaut à un cumul de connaissance et le rapport au savoir que l'élève peut développer est un rapport à un réel déjà décrit et structuré, existant hors de son champ de conscience. Ce rapport ne peut être que normatif et confirmatoire d'un rôle suprêmement important accordé à la fonction d'évaluation et de certification.

\section{Conclusion}

L'absence de distinction conceptuelle entre discipline et matière scolaire semble marquer l'univers scolaire, tant du point de vue des "organismes décisionnels " que des praticiennes et praticiens. Cette absence de distinction est lourde 
d'implications sur un double plan.

D'une part, l'identité conceptuelle établie entre matière scolaire et discipline scientifique implique que le processus d'enseignement ne serait qu'un processus de scolarisation du savoir. Ce dernier se définit d'ailleurs de façon exclusive en relation à l'ensemble des contenus et procédures définissant l'univers conceptuel, méthodologique, voire idéologique, propre à chaque champ scientifique, à chaque discipline scientifique homologuée ou reconnue. Bain (1997) décrit fort bien et de façon très explicite les dangers d'une telle position, notamment sur le plan de la pérennité du caractère éclaté, morcelé de ce qu'on désire présenter en tant que curriculum.

D'autre part, le même auteur (Bain, 1993, 1997) démontre aussi très clairement le risque de didactisation qui en résulte. La transposition ramenée à l'ajustement d'un discours scientifique ou techno-instrumental en fonction des caractéristiques développementales de l'enfant, ne correspond-elle pas à la logique même de la réorganisation des curricula vécue dans les années 1970 dans la perspective bloomienne? Si tel est le cas, pourquoi alors parler d'une réorientation constructiviste ou socioconstructiviste du curriculum dans le cadre d'une réforme qui met l'emphase sur le réagencement du temps alloué pour l'apprentissage des matières et, en conséquence, sur l'augmentation du temps accordé à l'exploration des matières dites essentielles ou principales?

Si la réalité existe indépendamment de notre perception et si l'expression de cette réalité se trouve déjà établie au travers des descriptions de ses composantes, dans le cadre des corpus de connaissances préalablement construites au sein des disciplines scientifiques reconnues, homologuées, pourquoi alors tenir compte des représentations initiales construites par les élèves hors du champ scolaire? Le rôle d'un curriculum "refondu " doit-il être celui d'une amplification de l'importance accordée aux moments d'exposition de l'élève à la vérité scientifique reformulée de sorte qu'elle soit accessible au «futur petit adulte en formation» et pondérée selon l'importance inégale accordée, semble-t-il, par l'ensemble des intervenantes et intervenants du primaire aux matières scolaires (Larose et Lenoir, 1998; Lebrun, Lenoir, Larose et Désilets, 1999)?

L'objet de cet article n'était pas tant de proposer des réponses que d'interroger la cohérence du discours gouvernemental au regard de certains concepts clés de l'actuelle réforme curriculaire. Certes, ce discours se veut porteur de modifications importantes, non seulement dans la façon de concevoir les finalités de l'apprentissage scolaire, mais aussi dans celle qui caractérise le statut de l'apprenant luimême. Ainsi ne peut-on que saluer la proclamation du virage « socioconstructiviste " qui caractérise semble-t-il le nouveau curriculum. Ce virage amène à concevoir que l'interaction entre apprenants peut être tout aussi porteuse de validation des savoirs construits que la confrontation de l'élève à une situation de performance dont le résultat sera évalué dans un contexte dont les paramètres lui demeurent parfaitement extérieurs. Néanmoins, nous croyons essentiel que le débat qu'engendre la réforme curriculaire en cours dépasse «l'innovation linguistique » et pose la question de la pertinence et du sens des concepts qui fondent l'acte d'enseigner lui-même. 
Transmet-on des connaissances, transpose-t-on des savoirs disciplinaires, agit-on dans un univers de connaissance différent de celui des disciplines de référence ou, si l'on préfère, des disciplines scientifiques, lorsqu'on intervient dans le champ de matières scolaires? Autant de questions auxquelles il importerait de répondre si l'on désire que la réforme de l'enseignement s'accompagne d'une réelle modification du rôle épousé et du rôle perçu des principaux acteurs du processus d'enseignement et d'apprentissage, en l'occurrence les acteurs d'un processus de médiation sociale entre construits sociaux (les savoirs homologués) et constructeurs de savoirs (les enseignants et les apprenants).

\section{Références bibliographiques}

ANGERS, P. et BOUCHARD, C. (1984). La mise en œuvre du projet intégration. Montréal: Bellarmin.

ANGERS, P. et BOUCHARD, C. (1990). Le jugement, les valeurs et l'action. Montréal: Bellarmin.

BAIN, D. (1993). Du bon usage des manuels : réflexions à partir de quelques expériences et analyses, dans J.-F. Ferret et E. Runtz-Christian (dir.), Les manuels font-ils école? Fribourg: Delval-IRDP, p. 31-49.

BAIN, D. (1997). La "scolarisation» du savoir : un dérapage inévitable de la transposition didactique? Éducation et recherche, vol. 19, n 1 , p. 8-28.

BEILLEROT, J. (1979). Le savoir, rapport et appropriation. Éducation permanente, vol. 47 , p. 45-51.

BEILLEROT, J. (1989). Le rapport au savoir : une notion en formation, dans J. Beillerot, A. Bouillet, C. Blanchard-Laville et N. Mosconi (dir.), Savoir et rapport au savoir. Élaborations théoriques et cliniques. Paris : Éditions universitaires, p. 165-202.

BERNSTEIN, B. (1971). On the Classification and Framing of Educational Knowledge, dans M.F.D. Young (dir.), Knowedge and Control. New Directions for the Sociology of Education. Londres: Collier-Macmillan, p. 47-69.

CONSEIL SUPÉRIEUR DE L'ÉDUCATION (1982). Le sort des matières dites «secondaires» au primaire - Avis au ministre de l'Éducation. Québec: Conseil supérieur de l'éducation.

CONSEIL SUPÉRIEUR DE L'ÉDUCATION (1998). Pour un renouvellement prometteur des programmes à l'école - Avis à la ministre de l'Éducation. Québec: Conseil supérieur de l'éducation. http://www.cse.gouv.qc.ca/f/pub/avis/avis.htm. 
CONSEIL SUPÉRIEUR DE L'ÉDUCATION (1999). Les enjeux majeurs des programmes d'études et des régimes pédagogiques - Avis à la ministre de l'Éducation. Québec: Conseil supérieur de l'éducation. http://www.cse.gouv.qc.ca/f/pub/avis/avis.htm.

DANDURAND, P. et OLLIVIER, É. (1991). Centralité des savoirs en éducation: vers de nouvelles problématiques. Sociologie et sociétés, vol. 23, nº 1, p. 3-23.

DE LANDSHEERE, G. (1979). Dictionnaire de l'évaluation et de la recherche en éducation. Paris : PUF.

GOODSON, I.F. (1987). School Subjects and Curriculum Change. Studies in Curriculum History. Londres : The Falmer Press.

GOODSON, I.F. (1997). The Changing Curriculum. Studies in Social Construction. New York (NY) : Peter Lang.

GOUVERNEMENT DU QUÉBEC (1996a). Les États généraux sur l'éducation; 1995-1996. Exposé de la situation. Québec : ministère de l'Éducation. http://www.meq.gouv.qc.ca/m_pub.htm.

GOUVERNEMENT DU QUÉBEC (1996b). Prendre le virage du succès. Plan d'action ministériel pour la réforme de l'éducation. Québec: ministère de l'Éducation. http://www.meq.gouv.qc.ca/reforme/reforme.htm\#2.

GOUVERNEMENT DU QUÉBEC (1997a). Réaffirmer l'école, prendre le virage du succès. Rapport du Groupe de travail sur la réforme du curriculum. Québec: ministère de l'Éducation. http://www.meq.gouv.qc.ca/publications/ menu-rapports.htm\#rapport.

GOUVERNEMENT DU QUÉBEC (1997b). Prendre le virage du succès. L'école tout un programme, énoncé de politique éducative. Québec : ministère de l'Éducation. http://www.meq.gouv.qc.ca/m_pub.htm.

GOUVERNEMENT DU QUÉBEC (1997c). Rapport d'activités du Comité d'orientation et de formation du personnel enseignant (COFPE). Québec : ministère de l'Éducation. http://www.meq.gouv.qc.ca/m_pub.htm.

GOUVERNEMENT DU QUÉBEC (1999). Orientations pour la formation continue du personnel enseignant. Choisir plutôt que subir le changement. Québec: ministère de l'Éducation. Direction de la formation et de la titularisation du personnel scolaire. http://www.meq.gouv.qc.ca/m_pub.htm.

LAFOREST, M. (1989). Diagnostic de l'enseignement des sciences humaines dans les classes primaires franco-catholiques du Québec (1959-1988). Paris : Université de Paris 7. Thèse de doctorat en sociologie de la connaissance (nouveau régime). 
LAROSE, F., AUDETTE, S. et ROY, G.-R. (1997a). La représentation sociale du français en formation professionnelle. Analyse de l'impact d'une rechercheaction-formation sur la représentation de l'utilité des compétences en français sur le plan de la formation et de la carrière. Éducation et Recherche, vol. 19, $\mathrm{n}^{\circ}$ 3, p. 349-373.

LAROSE, F., AUDETTE, S. et ROY, G.-R. (1997b). Analyse des représentations sociales des compétences linguistiques et technoprofessionnelles des étudiants du secondaire professionnel au Québec : aspects méthodologiques d'une nouvelle approche de l'étude de la motivation, dans R. Féger (Dir.), L'éducation face aux nouveaux défis. Montréal : Éditions Nouvelles AMS, p. 497-507.

LAROSE, F., JONNAERT, P. et LENOIR, Y. (1996a). Le construit de didactique : une étude lexicométrique illustrative d'un corpus de définitions. Éduquer et former, vol. 8, p. 28-44.

LAROSE, F., LAVALLÉE, M. et DEMERS, J. (1996b). Socialisation traditionnelle et éducation. L'acculturation et la représentation des rôles respectifs de la famille et de l'école en milieu inuit. Cahiers de la recherche en éducation, vol. $3, \mathrm{n}^{\circ} 2$, p. $239-270$.

LAROSE, F., LENOIR, Y. (1997). L'étude de l'impact d'un processus de formation continue sur les représentations sociales partagées par les enseignantes du primaire ainsi que sur leurs pratiques professionnelles en regard de l'interdisciplinarité pédagogique, dans R. Féger (dir.), L'éducation face aux nouveaux défis. Montréal : Éditions Nouvelles AMS, p. 146-157.

LAROSE, F. et LENOIR, Y. (1998). La formation continue d'enseignants du primaire à des pratiques interdisciplinaires : résultats de recherches. Revue des sciences de l'éducation, vol. 24, $\mathrm{n}^{\circ}$ 1, p. 189-228.

LAROSE, F., LENOIR Y. et KARSENTI, T. (à paraître). Construction sociale et construction individuelle du savoir. La genèse du savoir de sens commun et son rôle dans le développement conatif de l'individu. Cahiers internationaux de psychologie sociale.

LEBART, L., MORINEAU, A. et PIRON, M. (1995). Statistique exploratoire multidimensionnelle. Paris : Dunod.

LEBRUN, J., LENOIR, Y., LAROSE, F. et DÉSILETS, M. (1999). L'utilisation de matériaux didactiques par les enseignants du primaire: une approche interdisciplinaire. Contexte, problématique, objectifs et cadre théorique de la recherche. Sherbrooke: Université de Sherbrooke, Faculté d'éducation (Documents du GRIFE $n^{\circ}$ 7).

LEGENDRE, R. (1993). Dictionnaire actuel de l'éducation ( $2^{\mathrm{e}}$ édition). MontréalParis : Guérin/Eska.

LENOIR, Y. (1991). Relations entre interdisciplinarité et intégration des apprentissages dans l'enseignement des programmes d'études du primaire au Québec. Paris: Université de Paris 7. Thèse de doctorat (nouveau régime). 
LENOIR, Y. et LAROSE, F. (1999). Une typologie des pratiques interdisciplinaires chez les enseignants du primaire au Québec: résultats de différentes recherches. Sherbrooke: Université de Sherbrooke, Faculté d'éducation, Groupe de recherche sur l'interdisciplinarité dans la formation des enseignants (Documents du GRIFE $n^{0}$ 8).

LENOIR, Y., LAROSE, F. et GRENON, V. (à paraître). La hiérarchisation des matières scolaires chez les enseignants du primaire au Québec : évolution ou stabilité des représentations depuis 1981? Revue des sciences de l'éducation.

MARDIA, K., KENT, J. et BIBBY, J. (1979). Multivariate Analysis. San Diego : Academic Press.

MOLINER, P. (1995). A Two-dimensional Model of Social Representations. European Journal of Social Psychology, vol. 25, $\mathrm{n}^{\circ}$ 1, p. 27-40.

MOSCOVICI, S. (1984). Psychologie sociale. Paris : PUF.

MOSCOVICI, S. (1988). Notes Towards a Description of Social Representations. European Journal of Social Psychology, vol. 18, p. 211-250. 